\title{
MTA1 Aids the AKT Pathway by Inhibiting Expression of a Key Regulator, PTEN
}

\author{
Shimul Salot and Rajiv Gude* \\ Advanced Centre for Treatment, Research and Education in Cancer (ACTREC) Tata Memorial Centre, Sector 22, Kharghar, Navi Mumbai - 410 210, India
}

\begin{abstract}
Metastasis is the final result of actions of various genes, one of which is the Metastasis Associated 1 (MTA1) gene. MTA1 acts as part of a nucleosome remodeling and histone deacetylation complex and has been shown to aid metastasis by regulating many other molecules. We decided to study if there was any possible relationship between MTA1 and the phosphatase and tensin analogue mutated on chromosome 10 (PTEN). PTEN is a tumour suppressor gene known to be mutated in several cancers. We found that on knockdown of MTA1 using siRNA, PTEN protein levels increased albeit its mRNA levels were unchanged. We further found that MTA1 and PTEN colocalize and coimmunoprecipitate with each other. PTEN levels increased on inhibiting histone deacetyalse activity, such as possessed by MTA1. One of the most celebrated functions of PTEN is its regulation of the PI3K-Akt pathway. We found that the levels of active AKT decreased in cells treated with siRNA against MTA1. We hypothesize that MTA1 helps in maintaining the AKT pathway in cancer cells by inhibiting PTEN, a major antagonist of the pathway. This might be one of the several mechanisms by which MTA1 aids metastasis.
\end{abstract}

Keywords: Metastasis; MTA1; PTEN; PI3K; AKT; Histone deacetylation; Breast cancer

\section{Introduction}

Metastasis presents the toughest challenge in the treatment of cancer today. Although it is possible to reset the primary tumor, it is the spread of cancer cells that is difficult to target for treatment. Metastasis comprises of a series of events which include detachment of cells from the primary tumor, movement and invasion through the surrounding tissue, survival in the circulatory system and growth at the secondary site (Chambers et al., 2002). All these processes are governed through the cumulative effects of many genes and acquiring an understanding of the mechanism of actions of the products of such genes might enable us to devise better means of therapy against metastasis.

The metastasis associated 1 (MTA1) gene is one such gene which influences metastasis. MTA1 was identified through a differential cDNA library screening between high and low metastatic rat mammary adenocarcinoma cell lines (Toh et al., 1994; Toh et al., 1995). It is expressed at relatively low levels in most organs but a high expression is observed in testis, which is a site for extensive cellular proliferation and differentiation. Over expression of several genes has been shown to be important clinically (Pinto et al., 2008; Tsai et al., 2007). Expression of MTA1 has been shown to be upregulated in a variety of cancers, especially cancers of a highly invasive nature (Balasenthil et al., 2006; Kawasaki et al., 2008; Sasaki et al., 2002; Sasaki et al., 2001; Toh et al., 1999; Toh et al., 1997). High MTA1 expression has been related to high tumor grade and angiogenesis in breast cancer (Jang et al., 2006). High expression of MTA1 is also associated with increased relapse risk(Martin et al., 2006) and MTA1 has been mapped into a region showing significantly lower LOH (Martin et al., 2001) (loss of heterozygosity) in primary breast cancers with metastases compared to node-negative tumors, suggesting that epigenetic alterations of MTA1 affect metastatic potential. It has also been shown to contribute to drug resistance in premenopausal breast cancer patients through one of its target genes (Gururaj et al., 2006a). These facts have an immediate clinical importance.

MTA1 acts as part of the Nucleosome Remodeling and Deacetylation (NURD) complex (Xue et al., 1998). This complex is involved in remodeling of nuclesomes as well as deacetylation of histones, both processes known to affect global gene expression. Interestingly, MTA1 itself also has histone deacetylation activity(Yao and Yang, 2003). Many genes have been known to be regulated by MTA1 (Aramaki et al., 2005; Balasenthil et al., 2007; Gururaj et al., 2006b; Khaleque et al., 2008; Manavathi et al., 2007; Mishra et al., 2003; Talukder et al., 2004; Talukder et al., 2003). Although knowledge of its targeted genes and molecular partners continually emerges, the picture of its mechanism of actions in the cell is not entirely clear.

The phosphatase and tensin homologue deleted on chromosome ten (PTEN) is a tumor suppressor gene which is often deleted in malignant tumors (Li et al., 1997; Saal et al., 2008; Steck et al., 1997). It is noted most for its lipid phosphatase activity responsible for dephophorylating phosphatidylinositol (3,4,5)-triphosphate (PIP3) (Maehama and Dixon, 1998). PIP3 is an important secondary messenger that regulates many processes in the cell favouring metastasis. It recruits proteins containing the pleckstrin homology domain like the AKT family of proteins (Bellacosa et al., 1998). These phosphorylate a myriad of proteins eventually bringing about an increase in cellular proliferation, growth, angiogenesis, motility etc (Vivanco and Sawyers, 2002).

In this study we show that MTA1 regulates PTEN protein expression in two breast cancer cell lines. This led us to believe that both the proteins might interact with each other and we show that MTA1 and PTEN colocalize in the cell. The activity of the downstream target of PTEN, namely AKT, decreases on MTA1 knockdown.

*Corresponding author: Rajiv Gude, Gude lab, Advanced Centre for Treatment, Research and Education in Cancer (ACTREC), Tata Memorial Centre, Sector 22 , Kharghar, Navi Mumbai - 410210. INDIA, Tel: +912227405084; Fax: +912227412894; E-mail: rgude@actrec.gov.in

Received May 26, 2010; Accepted June 28, 2010; Published June 28, 2010

Citation: Shimul S, Rajiv G (2010) MTA1 Aids the AKT Pathway by Inhibiting Expression of a Key Regulator, PTEN. J Cancer Sci Ther 2: 114-119. doi:10.4172/1948-5956.1000034

Copyright: () 2010 Shimul Salot, et al. This is an open-access article distributed under the terms of the Creative Commons Attribution License, which permits unrestricted use, distribution, and reproduction in any medium, provided the original author and source are credited. 
Metastasis is complex process and this study might aid in further elucidating exactly at which steps MTA1 involves itself to regulate the spread of cancer.

\section{Materials and Methods}

\section{Reagents}

Cell lines were purchased from National Centre for Cell Science, INDIA. Cell culture media and serum were purchased from GIBCO, USA. Oligofectamine ${ }^{\mathrm{TM}}$ was purchased from Invitrogen, USA and siRNA from Ambion, USA. All SDS-PAGE reagents were purchased from Sigma Aldrich, USA. Primary antibodies against MTA1, PTEN, pan-Ac and Tubulin were purchased from Santacruz, USA and those against AKT and pAKT were purchased from Cell Signaling, USA. Anti-mouse and anti-rabbit horse raddish peroxidase conjugated secondary antibodies were purchased from Sigma. Trizol was purchased from Sigma and RT-PCR was carried out using kits from Fermentas, Canada. Anti-mouse FITC and anti-rabbit Alexa 568 secondary antibodies were purchased from Molecular probes, USA.

\section{Cell culture}

The human breast cancer cell lines MDA-MB-231 and MCF7 were grown in Dulbecco's Modified Eagle's medium (DMEM) supplemented with $10 \%$ heat inactivated fetal bovine serum (FBS) and antibiotics (100 U/ml penicillin, and $100 \mu \mathrm{g} / \mathrm{ml}$ streptomycin). The cell lines were maintained at $37^{\circ} \mathrm{C}$ in $5 \% \mathrm{CO} 2$ humidified atmosphere.

\section{Transfection}

Transfections were carried out according to manufacturer's instructions. Briefly, the day before transfection, cells were trypsinized and counted, and $0.3 \times 10^{6}$ cells were seeded in 6-well plates so that they were $50-60 \%$ confluent on the day of transfection. Cells were transfected using Oligofectamine ${ }^{\mathrm{Tm}}$. The reagent was diluted in medium without serum. The diluted reagent was incubated for 10 min at R.T. siRNA against MTA1 was used at 100nM concentration. The diluted Oligofectamine ${ }^{\mathrm{TM}}$ Reagent was added to diluted oligonucleotides, mixed gently, and incubated at room temperature for $20 \mathrm{~min}$. The mixture was overlayed onto the cells. Cells were incubated for $24 \mathrm{hrs}$ at $37^{\circ} \mathrm{C}$ in a $\mathrm{CO} 2$ incubator after which fresh media was added and cells were kept for a further $24 \mathrm{hrs}$. After 48 hours, cells were trypsinized and collected for further analysis. All transfections were carried out in media devoid of antibiotics. Two siRNAs targeting different exons were used the sequences of which were as follows:

\section{\#1:5'GCAUCAUUGAGUACUACUAtt3' 5'UAGUAGUACUCAAUGAUGCtg3' \\ \#2:5'CCUGCCCGAGAAACUAAAGtt3' 5'CUUUAGUUUCUCGGGCAGGtc3'}

\section{Western blotting}

Cells were harvested with $0.25 \%$ trypsin EDTA, centrifuged and lysed with lysis buffer solution containing $50 \mathrm{mM}$ Tris, $1 \%$ Triton X-100, 150mM NaCl, 5mM EDTA, 10mM EGTA, $50 \mathrm{mM}$ Na fluoride, $1 \mathrm{mM}$ Na orthovanadate, $1 \mathrm{mM}$ PMSF and protease inhibitor cocktail. Protein was estimated using Bradford reagent. Equal protein from all samples was loaded and separated on an $8 \%$ SDS-PAGE gel. The proteins were transferred to poly vinylidene difluoride (PVDF) membrane. Membranes were blocked for one hour using 5\% milk at room temperature (RT). Incubations with primary and secondary antibodies were carried out for one hour at RT. In case of detection of MTA1, direct incubation with primary antibody was performed overnight at $4^{\circ} \mathrm{C}$, followed by secondary antibody incubation as described earlier. The Pierce Femto chemiluminiscence detection system was used to detect signals.

\section{RT-PCR}

RNA was extracted by Trizol method. cDNA preparation and PCR were performed using kit from Fermentas according to manufacturer's instructions. Primers were manufactured by Sigma and had the following sequences:

PTEN: Forward, 5' - CCAAGTCCAGAGCCATTTC - 3'; Reverse, 5' GTGGGTCCTGAATTGGAGG - 3'.

MTA1: Forward, 5' - AAGCGCGGCTAACTTATT - 3'; Reverse, 5' GGCACCTTCAACAGAACACA-3'

GAPDH: Forward, 5'-GAGTCAACGGATTTGGTCGT - 3'; Reverse, 5' TGTGGTCATGAGTCCTTCCA - 3

\section{Coimmunoprecipitation}

Cells were grown in $90 \mathrm{~mm}$ plates for a period of 24 hours, trypsinized and collected. Cell lysates were prepared as mentioned in western blotting section. Agarose beads from Sigma were washed twice with washing buffer, centrifuged briefly at $12000 \mathrm{rpm}$ and resuspended in washing buffer. $10 \mu \mathrm{l}$ of primary antibody was added to tubes and the tubes were incubated for one hour on a shaker at RT. Samples were then washed and centrifuged and $200 \mu \mathrm{l}$ of cell lysate was added to each tube. These were incubated for 90 minutes on a shaker and then centrifuged. The pellet obtained was washed with washing buffer and loaded on SDS-PAGE gel. Further western blotting was carried out as described earlier. Non-specific antibody from Cell signaling was used as $\operatorname{IgG}$ control.

\section{Confocal analysis}

Cells were grown in $35 \mathrm{~mm}$ plates for a period of 24 hours to a confluency of $60-80 \%$ on coverslips (cell density was 1 X 106 on the day of the experiment). They were fixed in cold methanol for 3 minutes. They were permeabilized with $0.5 \%$ triton X-100 for 10 minutes and were blocked in 1\% BSA for half an hour. Incubation with the primary and secondary antibodies was carried out for one hour at room temperature. Coverslips were mounted on glass slides in $4 \%$ DABCO glycerol. Cells incubated with the flurochrome conjugated secondary antibodies alone (no primary antibody) were used as negative controls. Images were acquired at 63X using the LSM 510 META laser scanning microscope from Carl Zeiss, Germany. Images were analyzed using the software LSM 510.

\section{Results}

\section{MTA1 represses PTEN protein expression}

We used two breast cancer cell lines MDA-MB-231 and MCF7 cells to carry out all experiments. We used siRNA to downregulate MTA1 expression in these cell lines. Two different siRNAs targeting different exons of the MTA1 transcript were used at $100 \mathrm{nM}$ concentration. We checked the efficacy of the siRNAs by performing RT-PCR and western blotting analysis. At least 70\% knockdown of MTA1 protein was obtained using the siRNAs in both the cell lines (Supplementary Figure 1). Both mRNA and protein levels of MTA1 were reduced in siRNA treated cells (Figure 1). Transfected cells were checked for PTEN protein and mRNA expression. Western blots showed an increase in PTEN expression in siRNA (Figure 2) treated cells, indicating that MTA1 plays a role in the negative regulation of PTEN, a tumour suppressor gene. (Graphical presentations of the quantities 
of proteins with respect to tubulin are shown in supplementary Figure 2.) Interestingly, no such change was observed in the transcript levels of PTEN. It thus appears that MTA1 regulates PTEN only at the protein level in cancer cells.

\section{MTA1 physically associates with PTEN}

Since only protein but not mRNA levels of PTEN were affected in cells treated with siRNA against MTA1 we surmised that MTA1 and PTEN must be associated physically. Any physical association would render MTA1 to be in proximity with PTEN. We used confocal imaging to determine if MTA1 and PTEN colocalized in cells. Green signal is representative of MTA1 localization and red signals represent PTEN localization. As seen in Figure $3 a$ and Figure $3 b$, both proteins

A)

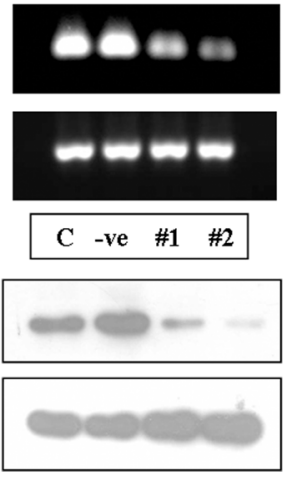

MTA1

B)

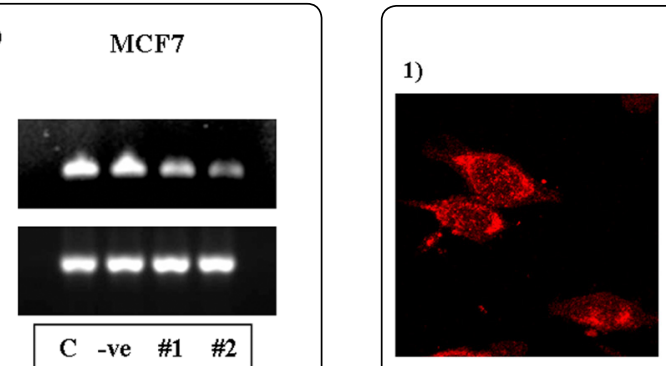

MTA1

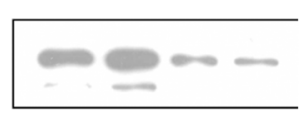

Tubulin

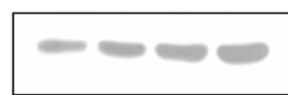

4)

C- untransfected cells, -ve - negative control siRNA, \#1 and \#2 - two siRNAs used against MTA1.

Figure 1: siRNA against MTA1 decreases its mRNA and protein expression. a) MDA-MB-231 and b) MCF7 cells were transfected with siRNAs against MTA1 or negative control siRNA and checked for expression of MTA1. Upper panel shows RT-PCR results and lower panel shows western blots. GAPDH and tubulin were used as loading controls.

A)

MDA-MB-231

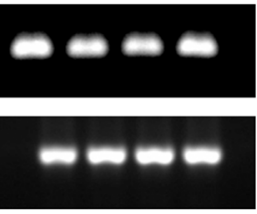

PTEN

B)

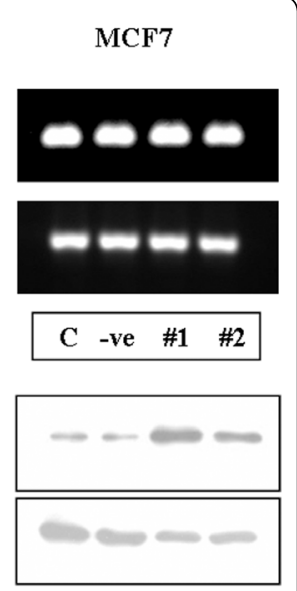

C- untransfected cells, -ve - negative control siRNA, \#1 and \#2 - two siRNAs used against MTA1.

Figure 2: PTEN protein expression is regulated by MTA1. a) MDA-MB-231 and b) MCF7 cells were transfected with siRNAs against MTA1 or negative control siRNA and checked for expression of PTEN. Upper panel shows RTPCR results and lower panel shows western blots. GAPDH and tubulin were used as loading controls. localize in the cytoplasm and the nucleus. Colocalization is indicated by a yellow signal. The presence of colocalization in the images was evaluated using LSM510 software and is shown in the form of the mask image. A mask is generated by the software itself and shows the exact areas in an image where colocalization occurs. In both the cell lines, it is clearly seen that MTA1 colocalizes with PTEN and this colocalization is more prominent near the nucleus.

In order to confirm the association between MTA1 with PTEN, we carried out co-immunoprecipation analysis. PTEN was immunoprecipitated from cell lysates. These precipitates were separated on a SDS-PAGE gel and transferred on membranes. PTEN immunoprecipiated complexes showed presence of MTA1, suggesting an interaction between the two molecules (Figure 4).

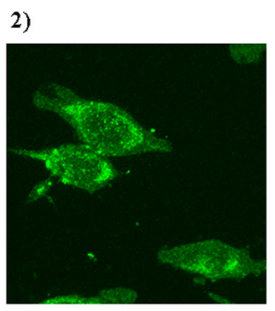

3)
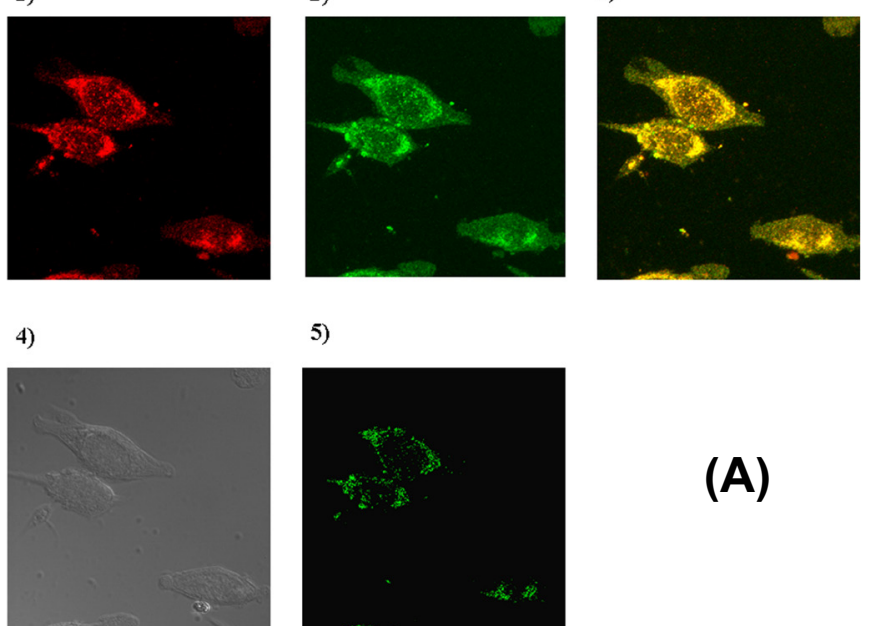

5)

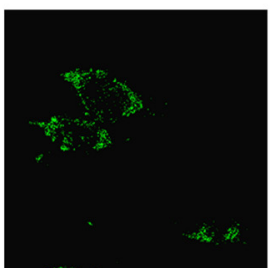

(A)

1)
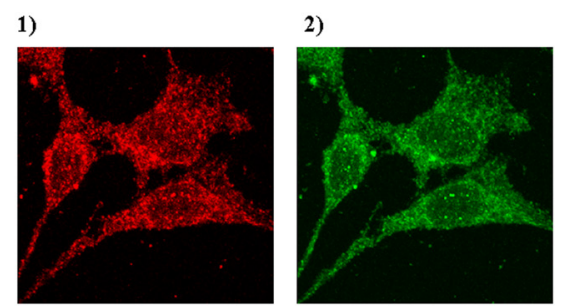

3)

4)

5)
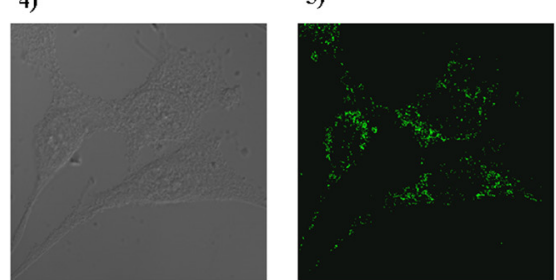

(B)

Figure 3: MTA1 and PTEN colocalize with each other a) MDA-MB-231 and b) MCF7 were fixed, permeabilized and incubated with antibodies against MTA1 and PTEN followed by incubation with fluorescently conjugated secondary antibodies. They were then subjected to confocal analysis. Images were acquired at 63X using the LSM 510 META laser scanning microscope from Carl Zeiss, Germany. Images were analyzed using the software LSM 510. 1) red signal indicates PTEN localization 2) green signal indicates MTA1 localization 3) yellow signal indicates colocalization 4) transmission image of cells 5) mask image. 


\section{PTEN expression is affected by deacetylation}

Since MTA1 functions as part of a complex involved in deacetylation, we treated both the cell lines with Trichostatin A (TSA), a histone deacetylase inhibitor, and checked PTEN levels. On TSA treatment, levels of PTEN increased (Figure 5a) indicating that PTEN expression can be affected by the process of deacetylation. We also studied the acetylation status of PTEN in the presence and absence of MTA1. For this purpose we immunoprecipitated cell lysates with anti-pan-Acetylation antibody, separated the lysates on an SDS-PAGE gel and carried out western blotting using anti-PTEN antibody. Levels of acetylated PTEN increased in absence of MTA1 in both the cell lines (Figure 5b).

\section{MTA1 affects PTEN activities in cells}

To test if events downstream of PTEN are also affected on MTA1 downregulation, we decided to choose a molecule downstream of PTEN activity and check its functionality. We chose AKT for this purpose. PTEN is mainly noted for its antagonistic behaviour towards the PI3K signaling pathway. PI3K regulates production of phosphatidylinositol $(3,4,5)$ trisphosphate which in turn activates the AKT kinase. AKT is

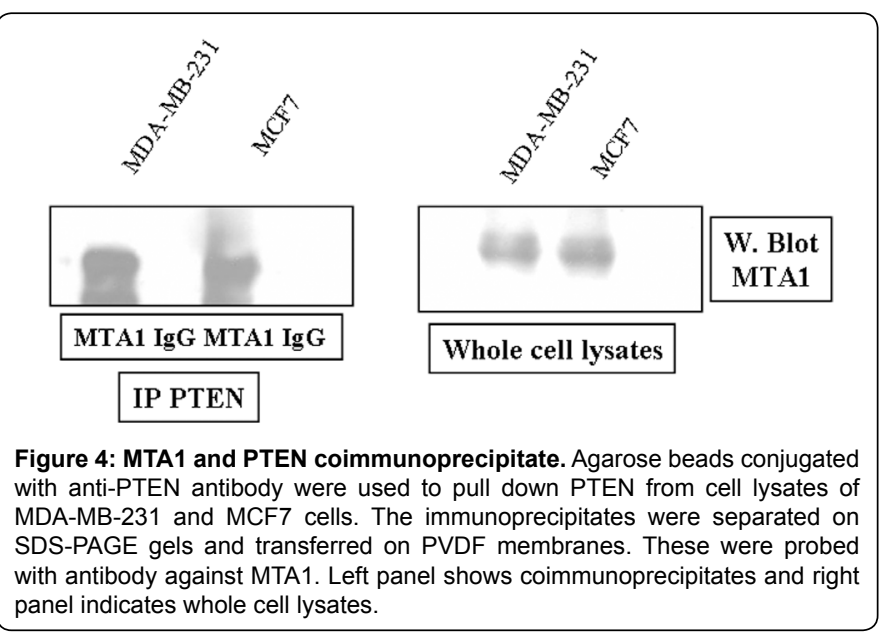

A)

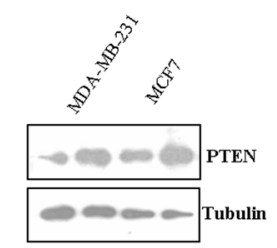

VC 100nM VC 100nM
B)

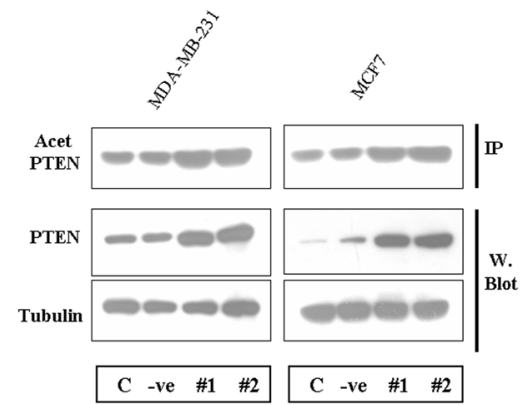

C- untransfected cells, -ve - negative control siRNA, \#1 and \#2 - two siRNAs used against MTA1.

Figure 5: PTEN expression is regulated by deacetylation. a) MDA-MB-231 and MCF7 cells were treated with Trichostatin A at a concentration of $100 \mathrm{nM}$. Cell lysates were subjected to western blotting. Blot shows PTEN expression. Ethanol was used as vector control as Trichostatin A is dissolved in ethanol. VC - vector control. b) MDA-MB-231 and MCF7 cells were transfected with siRNAs against MTA1 or negative control siRNA. Cell lysates from these samples were immunoprecipitated (IP) with anti-pan-Ac antibody, and then probed using anti-PTEN antibody. The expression of PTEN, and tubulin was analyzed by Western Blot analysis.$$
\text { a) }
$$

\section{MDA-MB-231}

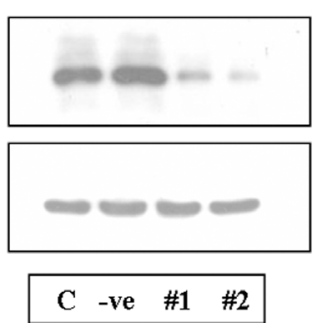

C- untransfected cells, -ve - negative control siRNA, \#1 and \#2 - two siRNAs used against MTA1

Figure 6: MTA1 regulates AKT activity. a) MDA-MB-231 and b) MCF7 cells were transfected with siRNAs against MTA1 or negative control siRNA and checked for expression of PAKT and AKT.

activated by the process of phosphorylation. We checked levels of phosphorylated AKT in MTA1 depleted cells. We used phosphor-AKT (Thr308) antibody which detects endogenous levels of AKT only when phosphorylated at threonine 308. Phosphorylated levels of AKT were reduced in MTA1 depleted cells as compared to control cells (Figure 6). Total AKT levels were unchanged. MTA1 thus appears to regulate the AKT pathway by regulating its upstream monitor, PTEN.

\section{Discussion}

Metastasis is a complex series of events, each of which is the product of cumulative inputs from many different molecules. It is the amalgamation of the actions of many genes which regulate the process of metastasis. To identify such genes which might play a favorable role in metastasis Toh et al. (1994) compared expression of transcripts by carrying out cDNA differential library screening of 2 rat mammary adenocarcinoma cell lines differing in their metastatic potential. One of the genes they identified was MTA1, the expression of which was higher in the highly metastatic cell line (Toh et al., 1994).

MTA1 was found to be associated with a higher metastatic potential in several cancers. Many players downstream of MTA1 have been identified. These in turn have some role or the other in pathways of the cell concerned with augmenting metastasis. Still, the list of molecules regulated by MTA1 is far from complete. We wanted to find out if PTEN could be added to this list.

PTEN is a dual-specificity phosphatase that has activity against lipid and protein substrates antagonistic towards the PI3K pathway. The phosphatidylinositol 3-kinase (PI3K) pathway regulates various cellular processes, such as proliferation, growth, apoptosis and cytoskeletal rearrangement. One of the main functions of PI3K is to synthesize the second messenger PtdIns $(3,4,5)$ P3 (PIP3) from PtdIns $(4,5)$ P2 (PIP2). AKT acts downstream of PI3K to regulate many biological processes (Vivanco and Sawyers, 2002).

We used siRNA to downregulate MTA1 expression in two breast cancer lines, MDA-MB-231 and MCF7, and observe if the depletion of MTA1 had any effect on PTEN expression. We did not observe any change in the transcript levels of PTEN. However, the protein levels of PTEN were altered in siRNA transfected cells. PTEN expression was increased in those cells with a reduced MTA1 expression. It is thus clear that MTA1 regulates PTEN expression by controlling its protein levels in the cell.

We surmised that MTA1 must interact with PTEN protein physically either directly or as part of a complex. We carried out 
coimmunoprecipitation analysis using PTEN antibody to pull down complexes containing PTEN and then probed blots with MTA1 antibody. We found that MTA1 co-immunprecipitated with PTEN in both MDA-MB-231and MCF7 lysates. We also checked if the interaction between these proteins could be visualized in cells. MTA1 and PTEN colocalized in both the cell lines. The colocalization is predominant near the nucleus and it is possible that MTA1 regulates PTEN protein primarily near this region. We plan to study this fact in future studies.

Since MTA1 is engaged in deacetylation, we tested if the change in expression of PTEN could be attributed to the process of deacetylation. We used trichostatin A, a known HDAC inhibitor to test if it had any effect on PTEN levels. TSA increased PTEN levels in both the cell lines. We also observed that levels of acetylated PTEN increased in siRNA transfected cells. However, it must also be noted that protein levels of PTEN themselves increase in siRNA transfected cells and hence any increase in the acetylated levels should be expected.

In order to confirm that the functionality of PTEN is also affected by MTA1, we decided to study the active AKT levels in cells, AKT being an important molecule downstream of PTEN regulation (Stambolic et al., 1998). AKT is recruited by PIP3 through interaction with its pleckstrin homology (PH) domain (Bellacosa et al., 1998). PIP3 also recruits another $\mathrm{PH}$-domain-containing serine/threonine kinase namely 3-phosphoinositide-dependent protein kinase-1 (PDK1). PDK1 phosphorylates AKT on Thr308 (Vanhaesebroeck and Alessi, 2000). Thr308 phosphorylation is necessary and sufficient for AKT activation (Stokoe et al., 1997); however, maximal activation requires additional phosphorylation at Ser473 by PDK2, another kinase (Alessi et al., 1997). Since the Thr308 phosphorylation is eventually controlled by PTEN activities, we checked levels of Thr308 phosphorylated AKT in MTA1 depleted cells. Active AKT was decreased in these cells as compared to control cells. Increase in PTEN levels in MTA1 depleted cells and the corresponding decrease in active AKT thus shows regulation of PTEN pathway by MTA1.

Proteasomal degradation of several proteins has been shown to be affected by the process of acetylation. (Caron et al., 2005) It is possible that MTA1 induces the proteasomal degradation of PTEN by influencing its acetylation. The fact that MTA1 is part of a deacetylation complex and that MTA1 physically associates with PTEN supports the idea that MTA1 deacetylates PTEN either alone or by recruiting the NURD complex which might induce PTEN degradation. We plan to study further, the consequences of regulation of PTEN by MTA1. MTA1 might be controlling several pathways in the cell through its action on PTEN. It would be interesting to see exactly which molecules downstream of PTEN are affected by the MTA1 pathway. Here we show functions of AKT being altered when MTA1 expression is disrupted. It is possible that pro-metastatic molecules like AKT receive aid from proteins like MTA1 to keep in check functions of their own negative regulators. Such regulations may be favoured by cancer cells turning metastatic. The final result of any series of changes in operations of such molecules is observed in the different events of the metastatic cascade. We plan to study exactly which processes in the cell get modified by MTA1's regulation of PTEN with the use of various functional assays.

MTA1 regulation of PTEN, a known tumor suppressor gene, is in synchronization with the generally accepted role of MTA1 as a gene helping to increase metastatic potential of cancer cells. An understanding of the mechanism of actions of metastatic favoring genes like MTA1 will eventually help us in understanding the disease itself and thus help us devise better therapies.

\section{Acknowledgements}

The study has been funded by the Department of Science and Technology (DST), Government of India.

\section{References}

1. Alessi DR, James SR, Downes CP, Holmes AB, Gaffney PR, et al. (1997) Characterization of a 3-phosphoinositide-dependent protein kinase which phosphorylates and activates protein kinase Balpha. Curr Biol 7: 261-269.

2. Aramaki $\mathrm{Y}$, Ogawa K, Toh Y, Ito T, Akimitsu N, et al. (2005) Direct interaction between metastasis-associated protein 1 and endophilin 3. FEBS Lett 579 : 3731-3736.

3. Balasenthil S, Broaddus RR, Kumar R (2006) Expression of metastasisassociated protein 1 (MTA1) in benign endometrium and endometrial adenocarcinomas. Hum Pathol 37: 656-661.

4. Balasenthil S, Gururaj AE, Talukder AH, Bagheri-Yarmand R, Arrington T, et al (2007) Identification of Pax5 as a target of MTA1 in B-cell lymphomas. Cancer Res 67: 7132-7138.

5. Bellacosa A, Chan TO, Ahmed NN, Datta K, Malstrom S, et al. (1998) Akt activation by growth factors is a multiple-step process: the role of the $\mathrm{PH}$ domain. Oncogene 17: 313-325.

6. Caron C, Boyault C, Khochbin S (2005) Regulatory cross-talk between lysine acetylation and ubiquitination: role in the control of protein stability. Bioessays 27: $408-415$

7. Chambers AF, Groom AC, MacDonald IC (2002) Dissemination and growth of cancer cells in metastatic sites. Nat Rev Cancer 2: 563-572.

8. Gururaj AE, Holm C, Landberg G, Kumar R (2006a) Breast cancer-amplified sequence 3 , a target of metastasis-associated protein 1 , contributes to tamoxifen resistance in premenopausal patients with breast cancer. Cell Cycle 5: 1407-1410.

9. Gururaj AE, Singh RR, Rayala SK, Holm C, den Hollander P, et al. (2006b) MTA1, a transcriptional activator of breast cancer amplified sequence 3. Proc Natl Acad Sci U S A 103: 6670-6675.

10. Jang KS, Paik SS, Chung H, Oh YH, Kong G (2006) MTA1 overexpression correlates significantly with tumor grade and angiogenesis in human breast cancers. Cancer Sci 97: 374-379.

11. Kawasaki G, Yanamoto S, Yoshitomi I, Yamada S, Mizuno A (2008) Overexpression of metastasis-associated MTA1 in oral squamous cell carcinomas: correlation with metastasis and invasion. Int J Oral Maxillofac Surg 37: 1039-1046.

12. Khaleque MA, Bharti A, Gong J, Gray PJ, Sachdev V, et al. (2008) Heat shock factor 1 represses estrogen-dependent transcription through association with MTA1. Oncogene 27: 1886-1893.

13. Li J, Yen C, Liaw D, Podsypanina K, Bose S, et al. (1997) PTEN, a putative protein tyrosine phosphatase gene mutated in human brain, breast, and prostate cancer. Science 275: 1943-1947.

14. Maehama T, Dixon JE (1998) The tumor suppressor, PTEN/MMAC1, dephosphorylates the lipid second messenger, phosphatidylinosito 3,4,5-trisphosphate. J Biol Chem 273: 13375-13378.

15. Manavathi B, Peng S, Rayala SK, Talukder AH, Wang MH, et al. (2007) Repression of Six3 by a corepressor regulates rhodopsin expression. Proc Nat Acad Sci USA 104: 13128-13133.

16. Martin MD, Fischbach K, Osborne CK, Mohsin SK, Allred DC, et al. (2001) Loss of heterozygosity events impeding breast cancer metastasis contain the MTA1 gene. Cancer Res 61: 3578-3580.

17. Martin MD, Hilsenbeck SG, Mohsin SK, Hopp TA, Clark GM, et al. (2006) Breas tumors that overexpress nuclear metastasis-associated 1 (MTA1) protein have high recurrence risks but enhanced responses to systemic therapies. Breast Cancer Res Treat 95: 7-12.

18. Mishra SK, Mazumdar A, Vadlamudi RK, Li F, Wang RA, et al. (2003) MICoA a novel metastasis-associated protein 1 (MTA1) interacting protein coactivator, regulates estrogen receptor-alpha transactivation functions. J Biol Chem 278 19209-19219. 
Citation: Shimul S, Rajiv G (2010) MTA1 Aids the AKT Pathway by Inhibiting Expression of a Key Regulator, PTEN. J Cancer Sci Ther 2: 114-119. doi:10.4172/1948-5956.1000034

19. Pinto M, Vieira J, Ribeiro FR, Soares MJ, Henrique R, et al. (2008) Overexpression of the mitotic checkpoint genes BUB1 and BUBR1 is associated with genomic complexity in clear cell kidney carcinomas. Cell Oncol 30: 389-395.

20. Saal LH, Gruvberger-Saal SK, Persson C, Lovgren K, Jumppanen M, et al. (2008) Recurrent gross mutations of the PTEN tumor suppressor gene in breast cancers with deficient DSB repair. Nat Genet 40: 102-107.

21. Sasaki H, Moriyama S, Nakashima Y, Kobayashi Y, Yukiue $H$, et al. (2002) Expression of the MTA1 mRNA in advanced lung cancer. Lung Cancer 35: 149154

22. Sasaki H, Yukiue H, Kobayashi Y, Nakashima Y, Kaji M, et al. (2001) Expression of the MTA1 mRNA in thymoma patients. Cancer Lett 174: 159-163.

23. Stambolic V, Suzuki A, de la Pompa JL, Brothers GM, Mirtsos C, et al. (1998) Negative regulation of PKB/Akt-dependent cell survival by the tumor suppressor PTEN. Cell 95: 29-39.

24. Steck PA, Pershouse MA, Jasser SA, Yung WK, Lin H, et al. (1997) Identification of a candidate tumour suppressor gene, MMAC1, at chromosome 10q23.3 that is mutated in multiple advanced cancers. Nat Genet 15: 356-362.

25. Stokoe D, Stephens LR, Copeland T, Gaffney PR, Reese CB, et al. (1997) Dual role of phosphatidylinositol-3,4,5-trisphosphate in the activation of protein kinase B. Science 277: 567-570.

26. Talukder AH, Gururaj A, Mishra SK, Vadlamudi RK, Kumar R (2004) Metastasisassociated protein 1 interacts with NRIF3, an estrogen-inducible nuclear receptor coregulator. Mol Cell Biol 24: 6581-6591.

27. Talukder AH, Mishra SK, Mandal M, Balasenthil S, Mehta S, et al. (2003) MTA1 interacts with MAT1, a cyclin-dependent kinase-activating kinase complex ring finger factor, and regulates estrogen receptor transactivation functions. J Biol Chem 278: 11676-11685.
28. Toh Y, Kuwano H, Mori M, Nicolson GL, Sugimachi K (1999) Overexpression of metastasis-associated MTA1 mRNA in invasive oesophageal carcinomas. $\mathrm{Br} \mathrm{J}$ Cancer 79: 1723-1726.

29. Toh Y, Oki E, Oda S, Tokunaga E, Ohno S, et al. (1997) Overexpression of the MTA1 gene in gastrointestinal carcinomas: correlation with invasion and metastasis. Int J Cancer 74: 459-463.

30. Toh Y, Pencil SD, Nicolson GL (1994) A novel candidate metastasisassociated gene, mta1, differentially expressed in highly metastatic mammary adenocarcinoma cell lines. cDNA cloning, expression, and protein analyses. J Biol Chem 269: 22958-22963

31. Toh Y, Pencil SD, Nicolson GL (1995) Analysis of the complete sequence of the novel metastasis-associated candidate gene, mta1, differentially expressed in mammary adenocarcinoma and breast cancer cell lines. Gene 159: 97-104.

32. Tsai WC, Chao YC, Sheu LF, Chang JL, Nieh S, et al. (2007) Overexpression of fascin-1 in advanced colorectal adenocarcinoma: tissue microarray analysis of immunostaining scores with clinicopathological parameters. Dis Markers 23 153-160.

33. Vanhaesebroeck B, Alessi DR (2000) The PI3K-PDK1 connection: more than just a road to PKB. Biochem J 346 Pt 3: 561-576.

34. Vivanco I, Sawyers CL (2002) The phosphatidylinositol 3-Kinase AKT pathway in human cancer. Nat Rev Cancer 2: 489-501.

35. Xue Y, Wong J, Moreno GT, Young MK, Cote J, et al. (1998) NURD, a novel complex with both ATP-dependent chromatin-remodeling and histone deacetylase activities. Mol Cell 2: 851-861.

36. Yao YL, Yang WM (2003) The metastasis-associated proteins 1 and 2 form distinct protein complexes with histone deacetylase activity. J Biol Chem 278: 42560-42568. 